\title{
Several Kinds of Chromatic Numbers of Multi-fan Graphs
}

\author{
Shunqin Liu \\ School of Information Science \& Technology, Xiamen University Tan Kah Kee College, Zhangzhou, China
}

Email address:

pytxlx@163.com

\section{To cite this article:}

Shunqin Liu. Several Kinds of Chromatic Numbers of Multi-fan Graphs. Applied and Computational Mathematics. Vol. 5, No. 3, 2016, pp. 133-137. doi: 10.11648/j.acm.20160503.16

Received: June 7, 2016; Accepted: July 8, 2016; Published: July 11, 2016

\begin{abstract}
Coloring problem is a classical difficult problem of graph theory. It is a fundamental problem in scientific computation and engineering design. In recent years, a variety of graph coloring problems frequently appeared and solved many problems in production. It is a difficult problem to discuss the chromatic number of a given graph class. In the paper, we introduce several kinds of chromatic numbers of graphs such as adjacent-vertex-distinguishing total chromatic number, adjacent-vertex-distinguishing proper edge chromatic number, smarandachely-adjacent-vertex-distinguishing edge chromatic number, and the multi-fan graphs are considered.
\end{abstract}

Keywords: Multi-fan Graphs, Adjacent-vertex-distinguishing Total Chromatic Number, Adjacent-vertex-distinguishing Proper Edge Chromatic Number, Smarandachely-adjacent-vertex-distinguishing Edge Chromatic Number

\section{Introduction}

Graph theory is an important branch of Applied Mathematics. Colouring problems originated in the four colour conjecture 150 years ago. In recent years, many interesting and useful results have been obtained on the study of colouring problems. It is widely used in chemistry, computer, communication and other fields. There for they are widely discussed in graph theory. In this paper, we introduce several kinds of chromatic numbers of graphs such as the adjacent -vertex -distinguishing total chromatic number, the adjacent -vertex-distinguishing proper edge chromatic number, the smarandachely-adjacent-vertex-distinguishing edge chromatic number. And the Multi-fan graphs are considered in this paper. In the end, the paper abtained the chromatic numbers of graphs the paper considered.

The graphs considered in this paper are connected, finite, undirected and simple graphs. The multi-fan graphs are joint graphs that jointed by $P_{1}$ and $P_{n_{1}} \cup P_{n_{2}} \cup \ldots \cup P_{n_{l}}$, which $P_{n_{k}}$ denotes the path graphs with order $n_{k}\left(n_{k} \geq 1\right)$. We denote for all $1 \leq k \leq l, V\left(P_{n_{k}}\right)=\left\{v_{1}{ }^{(k)}, v_{2}{ }^{(k)}, v_{n_{k}}{ }^{(k)}\right\}$ $v_{j}{ }^{(k)} v_{j+1}{ }^{(k)} \in E\left(P_{n_{k}}\right)\left(1 \leq j \leq n_{k}-1\right)$, and $P_{1}$ denotes the graph which has only one vertex $w$. The symbol $\Delta$ is the maximum degree of the graph we discussed.

The paper use apagoge, construction method and direct proving method.

\section{Adjacent-vertex-distinguishing Proper Total Coloring Number}

Defenition 1 [1] A k-proper total colouring of a graph $\mathrm{G}$ is a mapping $f$ from $V(G) \cup E(G)$ to $\{1,2, \ldots, k\}$ such that:

1) $\forall u, v \in V(G)$, if $u v \in E(G)$, then $f(u) \neq f(v)$;

2) $\forall e_{i}, e_{j} \in E(G), e_{i} \neq e_{j}$, if $e_{i}, e_{j}$ have a common end vertex, then $f\left(e_{i}\right) \neq f\left(e_{j}\right)$;

3) $\forall u \in V(G), e \in E(G)$, if $u$ is the end vertex of $e$, then $f(u) \neq f(e)$.

Let $f$ be a k-proper-total-colouring of $G$.Denote $C(u)=\{f(u)\} \bigcup\{f(u v) \mid v \in V(G) \wedge u v \in E(G)\}$ for every $u \in V(G)$, if $\forall u, v \in V(G), u v \in E(G)$, we have $C(u) \neq C(v) \quad, \quad$ then $f$ is called a k-proper-adjacent-vertex-distinguishing proper total coloring, short for k-AVDTC. 
The number $\min \{k \mid G$ has a k-proper-adjacent vertex-distinguishing total coloring $\}$ is called the adjacent vertex-distinguishing proper total chromatic number and denoted by $\chi_{a t}(G)$

The adjacent -vertex-distinguishing proper total chromatic number was first put forward by Zhang Zhong-fu, and he show a conjecture such that:

Conjecture 1 [11] For every connected graph $G$ with order at least 2 , we have $\chi_{a t}(G) \leq \Delta+3$.

Lemma 1 If two arbitrary distinct vertices of maximum degree in $G$ are not adjacent, then $\chi_{a t}(G) \geq \Delta+1$; If $G$ has two distinct vertices of maximum degree which are adjacent, then $\chi_{a t}(G) \geq \Delta+2$

Theorem 1: $\chi_{a t}\left(P_{1} \vee\left(P_{n_{1}} \cup P_{n_{2}}\right)\right)=n_{1}+n_{2}+1$

Proof. Because there is only one vertex $w$ whose degree $(=$ $n_{1}+n_{2}$ ) is the maximum degree, so concluded by Lemma 1 , we get the result such that

$$
\chi_{a t}\left(P_{1} \vee\left(P_{n_{1}} \cup P_{n_{2}}\right)\right) \geq n_{1}+n_{2}+1
$$

Then we let $f$ be a mapping from

$V\left(P_{1} \vee\left(P_{n_{1}} \cup P_{n_{2}}\right)\right) \cup E\left(P_{1} \vee\left(P_{n_{1}} \cup P_{n_{2}}\right)\right)$ to

$\left\{1,2,3, \ldots, n_{1}+n_{2}+1\right\}$ as follows :

$f(w)=n_{1}+n_{2}+1 \quad f\left(v_{n_{1}}{ }^{(1)}\right)=1 \quad f\left(v_{n_{2}}{ }^{(2)}\right)=n_{1}+1$

$f\left(v_{j}{ }^{(1)}\right)=j+1 \quad\left(1 \leq j \leq n_{1}-1\right)$

$f\left(v_{i}^{(2)}\right)=n_{1}+i+1 \quad\left(1 \leq i \leq n_{2}-1\right)$

$f\left(w v_{j}{ }^{(1)}\right)=j \quad\left(1 \leq j \leq n_{1}\right) \quad f\left(v_{1}^{(1)} v_{2}{ }^{(1)}\right)=n_{1}$

$f\left(w v_{i}^{(2)}\right)=n_{1}+i \quad\left(1 \leq j \leq n_{2}\right)$

$f\left(v_{1}^{(2)} v_{2}^{(2)}\right)=n_{1}+n_{2}$

$f\left(v_{j}{ }^{(1)} v_{j+1}{ }^{(1)}\right)=j \quad\left(2 \leq j \leq n_{1}-1\right)$

$f\left(v_{i}{ }^{(2)} v_{i+1}{ }^{(2)}\right)=n_{1}+i \quad\left(2 \leq i \leq n_{2}-1\right)$

At this time, we have

$C(w)=\left\{1,2,3, \ldots, \quad n_{1}, n_{1}+1, \ldots, \quad n_{1}+n_{2}+1\right\}$

$C\left(v_{1}{ }^{(1)}\right)=\left\{1,2, n_{1}\right\}$

$C\left(v_{2}{ }^{(1)}\right)=\left\{1,2,3, \quad n_{1}\right\}$

$C\left(v_{j}{ }^{(1)}\right)=\{j-2, j-1, j, j+1\} \quad\left(3 \leq j \leq n_{1}-1\right)$

$C\left(v_{n_{1}}{ }^{(1)}\right)=\left\{n_{1}-2, n_{1}, 1\right\}$

$C\left(v_{1}^{(2)}\right)=\left\{n_{1}+1, n_{1}+2, n_{1}+n_{2}\right\}$

$C\left(v_{2}{ }^{(2)}\right)=\left\{n_{1}+1, n_{1}+2, n_{1}+3, n_{1}+n_{2}\right\}$

$C\left(v_{i}^{(2)}\right)=\left\{n_{1}+i-2, n_{1}+i-1, n_{1}+i, n_{1}+i+1\right\}$

$$
\left(3 \leq i \leq n_{2}-1\right)
$$

$$
C\left(v_{n_{2}}{ }^{(2)}\right)=\left\{n_{1}+n_{2}-2, n_{1}+n_{2}, n_{1}+1\right\}
$$

As defined in definition 1, obviously, $f$ is a $n_{1}+n_{2}+1$ -AVDTC.

There for

$$
\chi_{a t}\left(P_{1} \vee\left(P_{n_{1}} \cup P_{n_{2}}\right)\right)=n_{1}+n_{2}+1
$$

Corollary 1

$$
\chi_{a t}\left(P_{1} \vee\left(P_{n_{1}} \cup P_{n_{2}} \cup \ldots \cup P_{n_{l}}\right)\right)=n_{1}+n_{1}+\ldots n_{l}+1
$$

The proof of Corollary 1 can be easy done.

\section{Adjacent-vertex-distinguishing Proper Edge Chromatic Number}

Defenition 2 [2] A k-proper-edge-colouring of a graph $\mathrm{G}$ is a mapping $f$ from $E(G)$ to $\{1,2, \ldots, k\}$ such that:

1) $\forall e_{i}, e_{j} \in E(G), e_{i} \neq e_{j}$, if $e_{i}, e_{j}$ have a common end vertex, then $f\left(e_{i}\right) \neq f\left(e_{j}\right)$;

Let $f$ be a k-proper-edge-colouring of $G$.Denote $C(u)=\{\{f(u v) \mid v \in V(G) \wedge u v \in E(G)\}$ for every $u \in V(G)$, if $\forall u, v \in V(G), u v \in E(G)$, we have $C(u) \neq C(v)$, then $f$ is called a k-proper-adjacentvertex-distinguishing-edge coloring, short for k-AVDPEC.

The number $\min \{k \mid G$ has a k-proper-adjacentvertex-distinguishing edge colouring $\}$ is called the adjacent vertex-distinguishing edge chromatic number and denoted by $\chi_{a}{ }^{\prime}(G)$.

For graphs $G, n_{i}$ denote the number of the vertex whose degree $=i, \delta, \Delta$ denote the minimum degree and the maximum degree of the graph. Then, define number $v(G)$ such that

$$
v(G)=\max \left\{\min \left\{\lambda \mid\left(\begin{array}{l}
\lambda \\
2
\end{array}\right) \geq n_{i}\right\}, \delta \leq i \leq \Delta\right\}
$$

Then a conjecture was put forward by [12]

Conjecture 2 For graphs without isolated edge and the number of the isolated vertex is no more than one, then

$$
v(G) \leq \chi_{a}{ }^{\prime}(G) \leq v(G)+1
$$

Lemma 2: For all graphs $G, \chi_{a}{ }^{\prime}(G) \geq \Delta$.

Theorem 2: $\chi_{a}{ }^{\prime}\left(P_{1} \vee\left(P_{n_{1}} \cup P_{n_{2}}\right)\right)=n_{1}+n_{2}$

Proof. There is only one vertex $w$ whose degree $(=$ $n_{1}+n_{2}$ ) is the maximum degree, so concluded by Lemma 2, we get the result such that

$$
\chi_{a}{ }^{\prime}\left(P_{1} \vee\left(P_{n_{1}} \cup P_{n_{2}}\right)\right) \geq n_{1}+n_{2}
$$

Then we let $f$ be a mapping from 
$E\left(P_{1} \vee\left(P_{n_{1}} \cup P_{n_{2}}\right)\right) \quad$ to $\quad\left\{1,2,3, \ldots, n_{1}+n_{2}\right\} \quad$ as follows :

$$
\begin{aligned}
& f\left(w v_{j}{ }^{(1)}\right)=j \quad\left(1 \leq j \leq n_{1}\right) \quad f\left(v_{1}^{(1)} v_{2}{ }^{(1)}\right)=n_{1} \\
& f\left(w v_{i}^{(2)}\right)=n_{1}+i \quad\left(1 \leq j \leq n_{2}\right) \\
& f\left(v_{1}^{(2)} v_{2}^{(2)}\right)=n_{1}+n_{2} \\
& f\left(v_{j}^{(1)} v_{j+1}{ }^{(1)}\right)=j \quad\left(2 \leq j \leq n_{1}-1\right) \\
& f\left(v_{i}^{(2)} v_{i+1}{ }^{(2)}\right)=n_{1}+i \quad\left(2 \leq i \leq n_{2}-1\right)
\end{aligned}
$$

At this time, we have

$$
\begin{aligned}
& C(w)=\left\{1,2,3, \ldots, \quad n_{1}, n_{1}+1, \ldots, n_{1}+n_{2}\right\} \\
& C\left(v_{1}{ }^{(1)}\right)=\left\{1, n_{1}\right\} \\
& C\left(v_{2}{ }^{(1)}\right)=\left\{1,2, n_{1}\right\} \\
& C\left(v_{j}{ }^{(1)}\right)=\{j-2, j-1, j\} \quad\left(3 \leq j \leq n_{1}-1\right) \\
& C\left(v_{n_{1}}{ }^{(1)}\right)=\left\{n_{1}-2, n_{1}\right\} \\
& C\left(v_{1}{ }^{(2)}\right)=\left\{n_{1}+1, n_{1}+n_{2}\right\} \\
& C\left(v_{2}{ }^{(2)}\right)=\left\{n_{1}+1, n_{1}+2, n_{1}+n_{2}\right\} \\
& C\left(v_{i}^{(2)}\right)=\left\{n_{1}+i-2, n_{1}+i-1, n_{1}+i\right\} \quad\left(3 \leq i \leq n_{2}-1\right) \\
& C\left(v_{n_{2}}{ }^{(2)}\right)=\left\{n_{1}+n_{2}-2, \quad n_{1}+n_{2}\right\}
\end{aligned}
$$

As defined in definition 2, obviously, $f$ is a $n_{1}+n_{2}$ -AVDPEC.

There for

$$
\chi_{a}^{\prime}\left(P_{1} \vee\left(P_{n_{1}} \cup P_{n_{2}}\right)\right)=n_{1}+n_{2}
$$

Corollary 2

$$
\chi_{a}{ }^{\prime}\left(P_{1} \vee\left(P_{n_{1}} \cup P_{n_{2}} \cup \ldots \cup P_{n_{l}}\right)\right)=n_{1}+n_{1}+\ldots n_{l}
$$
2.

The proof can be easy copied from the proof of Theorem

\section{Smarandachely}

\section{Adjacent-vertex-distinguishing Proper Edge Chromatic Number}

Defenition 3 [3] Let $f$ be a k-proper-edge-colouring of $G$.Denote $C(u)=\{\{f(u v) \mid v \in V(G) \wedge u v \in E(G)\}$ for every $u \in V(G)$, if $\forall u, v \in V(G), u v \in E(G)$, we have $C(u) \not \subset C(v)$ and $C(v) \not \subset C(u)$, then $f$ is called a smarandachely adjacent-vertex-distinguishing proper edge colouring, short for k-SA.

The number $\min \{k \mid G$ has a k smarandachely adjacentvertex-distinguishing proper edge coloring\} is called the smarandachely adjacent -vertex-distinguishing proper edge chromatic number and denoted by $\chi_{a s}{ }^{\prime}(G)$.

Lemma 3: If $G$ is a graph without one degree vertex, then $\chi_{\text {as }}{ }^{\prime}(G) \geq \Delta+1$.

Theorem 3:

i) If $n_{1} \equiv 0(\bmod 2)^{\text {and }} n_{2} \equiv 0(\bmod 2)$,

Then $\chi_{a s}{ }^{\prime}\left(P_{1} \vee\left(P_{n_{1}} \cup P_{n_{2}}\right)=n_{1}+n_{2}+1\right.$.

ii) If $n_{1} \equiv 1(\bmod 2)$ or $n_{2} \equiv 1(\bmod 2)$, then

$$
\chi_{\text {as }}{ }^{\prime}\left(P_{1} \vee\left(P_{n_{1}} \cup P_{n_{2}}\right)=n_{1}+n_{2}+2\right.
$$

Proof. i) Obviously, the maximum degree of $P_{1} \vee\left(P_{n_{1}} \cup P_{n_{2}}\right)$ denotes by $\Delta$, then $\Delta=n_{1}+n_{2}$,

so

$$
\chi_{a s}{ }^{\prime}\left(P_{1} \vee\left(P_{n_{1}} \cup P_{n_{2}}\right) \geq n_{1}+n_{2}+1\right.
$$

Then we give the mapping $f$ from

$$
E\left(P_{1} \vee\left(P_{n_{1}} \cup P_{n_{2}}\right)\right.
$$

to

$$
\left\{1,2, \ldots, \quad n_{1}+n_{2}\right\}
$$

as below:

$$
\begin{aligned}
& f\left(w v_{j}^{(1)}\right)=j \quad\left(1 \leq j \leq n_{1}\right) \\
& f\left(w v_{i}^{(2)}\right)=n_{1}+i \quad\left(1 \leq j \leq n_{2}\right)
\end{aligned}
$$$$
f\left(v_{2}{ }^{(1)} v_{3}{ }^{(1)}\right)=n_{1}+1
$$$$
f\left(v_{2 i-1}{ }^{(1)} v_{2 i}{ }^{(1)}\right)=n_{1}+n_{2}+1 \quad\left(1 \leq i \leq \frac{n_{1}}{2}\right)
$$$$
f\left(v_{2 i+2}{ }^{(1)} v_{2 i+3}{ }^{(1)}\right)=i \quad\left(1 \leq i \leq \frac{n_{1}}{2}\right)
$$$$
f\left(v_{2 i-1}{ }^{(2)} v_{2 i}{ }^{(2)}\right)=n_{1}+n_{2}+1 \quad\left(1 \leq i \leq \frac{n_{2}}{2}\right)
$$

$$
f\left(v_{2 i+2}^{(2)} v_{2 i+3}^{(2)}\right)=i \quad\left(1 \leq i \leq \frac{n_{2}}{2}\right)
$$

$f\left(v_{2 i+2}{ }^{(2)} v_{2 i+3}{ }^{(2)}\right)=n_{1}+i \quad\left(1 \leq i \leq \frac{n_{2}}{2}\right)$

$f\left(v_{2}^{(2)} v_{3}^{(2)}\right)=1$ 
By listing $C(u)$ of every vertex of the graph, we can see that the mapping we give is a smarandachely adjacent-vertex-distinguishing proper edge coloring of $P_{1} \vee\left(P_{n_{1}} \cup P_{n_{2}}\right)$, so

$$
\chi_{a s}{ }^{\prime}\left(P_{1} \vee\left(P_{n_{1}} \cup P_{n_{2}}\right)=n_{1}+n_{2}+1\right.
$$

ii) First, we must illustrate that there have no $n_{1}+n_{2}+1$

-SA for graph $P_{1} \vee\left(P_{n_{1}} \cup P_{n_{2}}\right)$.

Assume that $n_{1} \equiv 1(\bmod 2)$ :

Suppose that $n_{1}+n_{2}+1$-SA for graph $P_{1} \vee\left(P_{n_{1}} \cup P_{n_{2}}\right)$ exist. And $|C(w)|=n_{1}+n_{2}$, so we denote $C(w)=\left\{1,2,3, \ldots, \quad n_{1}+n_{2}\right\}, f\left(w v_{j}{ }^{(1)}\right)=j$,

$$
f\left(w v_{i}^{(2)}\right)=n_{i}+i \text {. }
$$

Because for all $i, j$,

$$
c\left(v_{j}^{(1)}\right) / c(w) \geq 1 c\left(v_{i}^{(2)}\right) / c(w) \geq 1,
$$

$n_{1}+n_{2}+1 \in c\left(v_{j}^{(1)}\right) \quad\left(1 \leq j \leq n_{1}\right)$, there is $n_{1}$ times for $c\left(v_{j}{ }^{(1)}\right)$, but $n_{1}$ is and odd number, the result is a contradiction to handshaking lemma.

So $\chi_{a s}{ }^{\prime}\left(P_{1} \vee\left(P_{n_{1}} \cup P_{n_{2}}\right) \geq n_{1}+n_{2}+2\right.$.

Then we define a mapping from $E\left(P_{1} \vee\left(P_{n_{1}} \cup P_{n_{2}}\right)\right)$ to $\left\{1,2, \ldots, n_{1}+n_{2}+2\right\}$ like this:

$$
\begin{aligned}
& f\left(w v_{j}{ }^{(1)}\right)=j \quad\left(1 \leq j \leq n_{1}\right) \\
& f\left(w v_{i}^{(2)}\right)=n_{1}+i \quad\left(1 \leq j \leq n_{2}\right) \\
& f\left(v_{2 i-1}{ }^{(1)} v_{2 i}{ }^{(1)}\right)=n_{1}+n_{2}+1 \quad\left(1 \leq i \leq\left[\frac{n_{1}}{2}\right]\right) \\
& f\left(v_{2 i}{ }^{(2)} v_{2 i+1}{ }^{(2)}\right)=n_{1}+n_{2}+2 \quad\left(1 \leq i \leq \frac{n_{1}}{2}\right) \\
& f\left(v_{2 i-1}{ }^{(2)} v_{2 i}{ }^{(2)}\right)=n_{1}+n_{2}+1 \quad\left(1 \leq i \leq\left[\frac{n_{2}}{2}\right]\right) \\
& f\left(v_{2 i}{ }^{(2)} v_{2 i+1}{ }^{(2)}\right)=n_{1}+n_{2}+2 \quad\left(1 \leq i \leq \frac{n_{2}}{2}\right)
\end{aligned}
$$

By listing $C(u)$ of every vertex of the graph, we can see that the mapping we give is a smarandachely adjacent-vertex-distinguishing proper edge colouring of $P_{1} \vee\left(P_{n_{1}} \cup P_{n_{2}}\right)$.
So $\chi_{a s}{ }^{\prime}\left(P_{1} \vee\left(P_{n_{1}} \cup P_{n_{2}}\right)=n_{1}+n_{2}+2\right.$.

\section{Conclusion}

Through the paper's research, conclusions are follows:

Theorem 1: $\chi_{a t}\left(P_{1} \vee\left(P_{n_{1}} \cup P_{n_{2}}\right)\right)=n_{1}+n_{2}+1$

Theorem 2: $\chi_{a}{ }^{\prime}\left(P_{1} \vee\left(P_{n_{1}} \cup P_{n_{2}}\right)\right)=n_{1}+n_{2}$

Theorem 3:

i) If $n_{1} \equiv 0(\bmod 2)$ and $n_{2} \equiv 0(\bmod 2)$,

Then $\chi_{a s}{ }^{\prime}\left(P_{1} \vee\left(P_{n_{1}} \cup P_{n_{2}}\right)=n_{1}+n_{2}+1\right.$

ii) If $n_{1} \equiv 1(\bmod 2)$ or $n_{2} \equiv 1(\bmod 2)$, then

$$
\chi_{a s}{ }^{\prime}\left(P_{1} \vee\left(P_{n_{1}} \cup P_{n_{2}}\right)=n_{1}+n_{2}+2\right.
$$

Theorem 1 and Theorem 2 are consistent with the Conjecture 1 and Conjecture 2. Then in future we can also study the upper limit of the smarandachely adjacent vertex-distinguishing proper edge chromatic number.

\section{References}

[1] Chen Xiang-en, Zhang Zhong-fu, "Adjacent-Vertex-Distinguishing Total Chromatic Number of $P_{m} \times K_{n}$," Journal of Mathematical Reserch and Exposition, Dalian. vol. A26, pp. 489-494, August 2015.

[2] Liu Hua, Ye Jian-hua, "Adjacent Vertex-Distinguishing Edges Coloring of ( $S_{m} * S_{n}$ )" Journal of East China Jiaotong University. vol 24. pp. 157-158, October 2007.

[3] Liu Shun-qin, Chen Xiang-en. "Smarandachely Adjacent-vert -ex-distinguishing Proper Edge Coloring of $\bar{K}_{m} \vee K_{n}$ ". Journal of Lanzhou University of Technology. vol. 41. pp. 155-158, August 2015.

[4] Zhang Dong-han, Zhang Zhong-fu. "The Upper Bound of the Adjacent Vertex Strongly Distinguishing Total Chromatic Number of the Graph". Advances in Mathematics. vol. 40 pp. 168-172. April 2011.

[5] Qiang Hui-ying, Li Mu-chun. "A Bound on Vertex Distinguishing Total Coloring of Graphs with Distance Constrant for Recurrent Event Data". Acta Mathematicae Applicatae Sinica. vol. 34. pp. 554-559. May 2011.

[6] Tian Jing-jing, Deng Fang-an. “Adjacent Vertex-distinguishing VE-Total Chromatic Number of the Crown Graph $C_{m} \bullet F_{n}$ and $C_{m} \bullet C_{n}$ ". Mathematics in Practice and Theory. vol. 41 . pp. 189-192. August. 2011.

[7] Yao Bing, Cheng Hui. "Behaviors of Vertex Neighbors of Trees Under Some Graph Coloring”. AclaMathematica Scientia. vol. 31. pp. 567-576. May 2011 
[8] Wen Fei, Wang Zhi-wen. "Vertex -distinguishing Total Coloring of Some Complement Double Graphs'. Journal of Shandong University (Natural Science). vol. 46. pp. 45-50 February 2011.

[9] Chen Xiang-en, Ma Yan-rong. “Adjacent-Vertex-Distingui -shing Total Chromatic Number of $K_{r} \vee K_{s}$ ". Journal of Jilin University (Science Edition). vol. 49. pp. 68-70. January 2011 .

[10] Tian Jing-jing. "The Smarandachely Adjacent -Vertex -Eege
Coloring of Some Mycielski's Graph". Journal of Math(PRC). vol. 32. pp. 723-728. April 2012.

[11] Zhang Zhong-fu, Chen Xiang-en, Li Jing-wen. "On adjacent vertex-distinguishing total coloring of graphs." Sci.China. Ser vol. 48. pp. 289-299. June 1997.

[12] Li Jing-wen, $\mathrm{Xu}$ Ban-gen, $\mathrm{Li}$ Mu-chun. "On the vertex-distinguishing chromatic number of $P_{m} \vee C_{n}$ " Journal of Shan Dong University (Nature Science ). vol. 43. pp. 24-30 August 2008. 